$\underline{\text { Article }}$

\title{
Synthesis of 3-Substituted 1,4-Benzodiazepin-2-ones
}

\author{
Kyungjin Kim, Steven K. Volkman, and Jonathan A. Ellman* \\ Department of Chemistry, University of California, Berkeley, California 94720
}

Received: August 10, 1998

\begin{abstract}
A preparação de benzodiazepínicos 1,4 substituídos na posição 3 é explorada a partir da alquilação de enolatos benzodiazepínicos. Empregando-se tal abordagem, vários gramas de benzodiazepínicos 1 foram preparados para estudos em animais, visando avaliar uma nova abordagem no tratamento da doença auto-imune lúpus eritematoso (LE).
\end{abstract}

The preparation of 3-substituted 1,4-benzodiazepines by benzodiazepine enolate alkylation has been explored. Employing this approach, multigram quantities of benzodiazepine $\mathbf{1}$ have been prepared for animal studies to evaluate a new approach for the treatment of the autoimmune disease systemic lupus erythematosus (SLE).

Keywords: benzodiazepine enolate-alkylation, systemic lupus erythematosus

\section{Introduction}

Benzodiazepines are one of the most important classes of therapeutic agents. For example, different benzodiazepines have anxiolytic, anticonvulsant and antihypnotic activities $^{1}$, serve as cholecystokinin A and B antagonists ${ }^{2}$, opioid receptor ligands ${ }^{3}$, platelet-activating factor antagonists $^{4}$, HIV trans-activator Tat antagonists ${ }^{5}$, HIV reverse transcriptase inhibitors ${ }^{6}$ and ras farnesyltransferase inhibitors $^{7}$. Due to the biological importance of benzodiazepines, we have carried out the solid-phase synthesis of libraries of over 10,000 unique 1,4-benzodiazepines derivatives ${ }^{8-12}$. These libraries have been assayed against a number of receptor and enzyme targets. In one study, benzodiazepine 1 was identified as the first small molecule inhibitor of autoantibody $\bullet$ DNA interactions in lupus-prone mice ${ }^{13}$. Related autoantibody $\bullet$ DNA interactions have been implicated in the autoimmune disease systemic lupus<smiles>CN1C(=O)C(Cc2ccc3ccccc3c2)N=C(c2ccc(O)cc2)c2cc(Cl)ccc21</smiles>

erythematosus (SLE) ${ }^{14}$. Blocking this interaction could potentially provide the first effective treatment of SLE. In order to perform animal studies to evaluate this potential strategy for treatment, large quantities of benzodiazepine $\mathbf{1}$ were required. Herein, we report an efficient synthesis route to multigram quantities of $\mathbf{1}$ and further describe the scope and limitations of this approach for the preparation of other 3-substituted benzodiazepine derivatives.

\section{Results and Discussion}

Most synthesis routes to 3-substituted benzodiazepin2-ones rely on the incorporation of amino acids into the benzodiazepine structure ${ }^{1,15}$. Accordingly, benzodiazepine 1 can be prepared from the nonproteinogenic amino acid $\beta$-naphthylalanine. However, due to the high cost of $\beta$ naphthylalanine, an alternative route was prefered for the preparation of large quantities of $\mathbf{1}$. Alkylation of the enolate of benzodiazepine 4 (Scheme 1) with 2-naphthylmethyl bromide could potentially provide a cost effective route to 1. Although Sternbach has documented the propensity for 1,4-benzodiazepines enolates to rearrange to isoindols ${ }^{16}$, other researchers in limited reports have described successful benzodiazepine enolate alkylations. ${ }^{17,18}$ In order to explore this approach, benzodiazepine $\mathbf{4}$ was prepared in three steps from aminobenzophenone $\mathbf{2}^{19}$. Treatment of $\mathbf{2}$ with bromoacetyl bromide in diethyl ether followed by amination and cyclization under acidic conditions provided 3 in $73 \%$ overall yield (Scheme 1). ${ }^{1,20} \mathrm{~N}$-Methylation with 


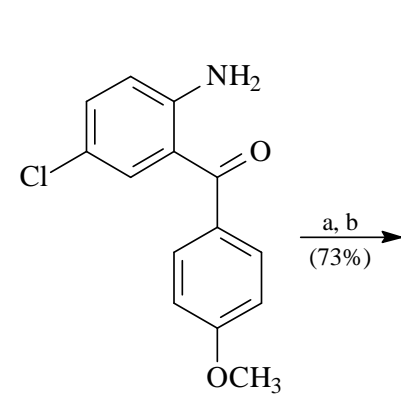

2<smiles>COc1ccc(C2=NCC(=O)Nc3ccc(Cl)cc32)cc1</smiles>

3<smiles>COc1ccc(C2=NCC(=O)N(C)c3ccc(Cl)cc32)cc1</smiles>

4

Scheme 1. (a) bromoacetyl bromide, diethyl ether, $0{ }^{\circ} \mathrm{C}$; (b) i. $\mathrm{NH}_{3} / \mathrm{MeOH}$, diethyl ether, rt, ii. $5 \%$ HOAc in tert-butyl alcohol, $50{ }^{\circ} \mathrm{C}$, $15 \mathrm{~h}$; (c) $\mathrm{K}_{2} \mathrm{CO} 3$, $\mathrm{CH}_{3} \mathrm{I}, \mathrm{MeOH} / \mathrm{THF}(1: 1)$, rt.

methyl iodide using potassium carbonate as a base then provided benzodiazepine 4 in $90 \%$ yield.

Next, we turned our attention to enolate alkylation of $\mathbf{4}$. Reaction conditions were optimized by evaluating a number of bases, reaction temperatures, and reagent stoichiometries. Benzodiazepine 5a (Table 1) was obtained in good yield (78\%) using potassium tert-butoxide as base with $\mathrm{THF}$ as solvent at $-78{ }^{\circ} \mathrm{C}$ (Method A). In order to evaluate the generality of these reaction conditions, several other benzodiazepine derivatives were also prepared (Table 1). Notably, with less reactive alkylating agents (entries 5d-5g), potassium bis(trimethylsilyl)amide should be used as the base (Method B). Under these conditions, moderate yields of the benzodiazepine products are observed.

Demethylation of 5a using aluminum tribromide in ethanethiol $^{21}$ proceeded in $86 \%$ yield, thereby providing benzodiazepine 1 . Greater than $50 \mathrm{~g}$ of benzodiazepine 1 has been prepared by this synthesis sequence.

\section{Conclusion}

Multigram quantities of benzodiazepine 1 have efficiently been prepared with the key step being enolate alkylation of benzodiazepine 4 . The scope and generality of preparing substituted benzodiazepines by enolate alkylation has also been established.<smiles>[R]C1N=C(c2ccc(OC)cc2)c2cc(Cl)ccc2N(C)C1=O</smiles>

Table 1. Enolate alkylation of benzodiazepine 4 .

\begin{tabular}{llcc}
\hline Entry $^{\mathrm{a}}$ & \multicolumn{1}{c}{ RX } & ${\text { Yield }(\%)^{\mathrm{b}}}^{\text {Rxn conditions }^{\mathrm{c}}}$ \\
\hline $5 \mathrm{a}$ & 2-(Bromomethyl)naphthalene & 78 & $\mathrm{~A}$ \\
$5 \mathrm{~b}$ & Benzyl bromide & 72 & $\mathrm{~A}$ \\
$5 \mathrm{c}$ & Iodomethane & 98 & $\mathrm{~A}$ \\
$5 \mathrm{~d}$ & Allyl bromide & 66 & $\mathrm{~B}$ \\
$5 \mathrm{e}$ & Ethyl bromide & 62 & $\mathrm{~B}$ \\
$5 \mathrm{f}$ & 1-Bromo-2-methylpropane & 39 & $\mathrm{~B}$ \\
$5 \mathrm{~g}$ & (Bromomethyl)cyclohexane & 48 & $\mathrm{~B}$ \\
\hline
\end{tabular}

${ }^{\mathrm{a}}$ All products were fully characterized by ${ }^{1} \mathrm{H}-\mathrm{NMR},{ }^{13} \mathrm{C}-\mathrm{NMR}$, and HRMS analysis. ${ }^{\mathrm{b}}$ Yields of pure compounds after chromatography. ${ }^{\mathrm{c}}$ See experimental section for reaction procedures. 


\section{Experimental}

\section{General}

Unless otherwise noted, materials were obtained from commercial suppliers and used without further purification. Tetrahydrofuran and diethyl ether were distilled under $\mathrm{N}_{2}$ from sodium/benzophenone immediately prior to use. Flash column chromatography was carried out using Merck 60 230-400 mesh silica gel. ${ }^{1} \mathrm{H}-\mathrm{NMR}$ spectra were obtained with a University of California at Berkeley Bruker AM-400 or AM-500 FT spectrometer. Proton-decoupled ${ }^{13} \mathrm{C}$-spectra were obtained at 100 or $125 \mathrm{MHz}$ with the same instruments. Chemical shifts are reported in ppm. High resolution mass spectra were obtained at the University of California at Berkeley mass spectrometry laboratory using fast atom bombardment (FAB) with 3-nitrobenzyl alcohol as matrix solvent.

\section{7-Chloro-5-(4-methoxyphenyl)-1,4-benzodiazepin-2-one}

To a solution of 2-amino-5-chloro-4'-methoxybenzophenone $2(12 \mathrm{~g}, 46 \mathrm{mmol})$ in diethyl ether $(200 \mathrm{~mL})$ at $0{ }^{\circ} \mathrm{C}$ were added bromoacetyl bromide $(5.2 \mathrm{~mL}, 60 \mathrm{mmol})$ and about $50 \mathrm{~g}$ of ice in one portion. After $1 \mathrm{~h}$ the white precipitate was filtered and washed with cold diethyl ether ( $3 \times 50 \mathrm{~mL}$ ) to afford the $\alpha$-bromoacetamide intermediate. Without further purification, the white powder was dissolved in a mixture of $100 \mathrm{~mL}$ of diethyl ether and $700 \mathrm{~mL}$ of a $13 \%$ (w./w.) solution of ammonia in methanol. After stirring overnight the solution was concentrated in vacuo to dryness. The white residue was dissolved in $300 \mathrm{~mL}$ of $10 \%$ acetic acid in tert-butanol and the reaction solution was heated to $60{ }^{\circ} \mathrm{C} 15 \mathrm{~h}$. The acidic solution was concentrated in vacuo. The residue was diluted with $500 \mathrm{~mL}$ of ethyl acetate, filtered through a silica gel pad, and concentrated in vacuo. The white powder was recrystallized from methanol to provide $10 \mathrm{~g}$ of product ( $73 \%$ yield), mp 198-199 ${ }^{\circ} \mathrm{C}:{ }^{1} \mathrm{H}-\mathrm{NMR}\left(500 \mathrm{MHz}, \mathrm{CDCl}_{3}\right) \delta 10.1(\mathrm{~s}, 1 \mathrm{H})$, $7.47(\mathrm{dt}, J=9,3 \mathrm{~Hz}, 2 \mathrm{H}), 7.42(\mathrm{dd}, J=8.5,2.5 \mathrm{~Hz}, 1 \mathrm{H})$, $7.31(\mathrm{~d}, J=2.5 \mathrm{~Hz}, 1 \mathrm{H}), 7.15(\mathrm{~d}, J=8.5 \mathrm{~Hz}, 1 \mathrm{H}), 6.89(\mathrm{dt}$, $J=9,3 \mathrm{~Hz}, 2 \mathrm{H}), 4.19-4.35$ (br s, 2H), 3.83 (s, 3H). ${ }^{13} \mathrm{C}-\mathrm{NMR}\left(125 \mathrm{MHz}, \mathrm{CDCl}_{3}\right) \delta 172.4,169.1,161.6,137.5$, 131.7, 131.3, 131.2, 130.7, 128.6, 122.8, 113.7, 56.4, 55.4. LRMS (FAB) m/e $301(\mathrm{M}+\mathrm{H})^{+}$; HRMS (FAB) $m / e$ 301.0744 [(M+H) $)^{+}$, calcd for $\mathrm{C}_{16} \mathrm{H}_{14} \mathrm{O}_{2} \mathrm{~N}_{2} \mathrm{Cl}$ : 310.0749].

\section{7-Chloro-5-(4-methoxyphenyl)-1-methyl- 1,4-benzodiazepin-2-one}

To a solution of $3(16 \mathrm{~g}, 53.2 \mathrm{mmol})$ in $160 \mathrm{~mL}$ of methanol-THF (1:1) were added potassium carbonate (44 $\mathrm{g}, 318 \mathrm{mmol})$ and iodomethane $(10 \mathrm{~mL}, 161 \mathrm{mmol})$. After stirring overnight the mixture was filtered through a Celite pad and concentrated in vacuo to afford $15 \mathrm{~g}$ of 4 (90\%) as a white solid: mp 68-70 ${ }^{\circ} \mathrm{C} ;{ }^{1} \mathrm{H}-\mathrm{NMR}\left(500 \mathrm{MHz}, \mathrm{CDCl}_{3}\right.$ ) $\delta 7.54(\mathrm{dt}, J=9,3 \mathrm{~Hz}, 2 \mathrm{H}), 7.48(\mathrm{dd}, J=9,2.5 \mathrm{~Hz}, 1 \mathrm{H})$, 7.26-7.29 (m, 2H), 6.89 (dt, $J=9,3 \mathrm{~Hz}, 2 \mathrm{H}), 4.74(\mathrm{~d}, J=$ $10.9 \mathrm{~Hz}, 1 \mathrm{H}), 3.82$ (s, 3H), 3.71 (d, $J=10.9 \mathrm{~Hz}, 1 \mathrm{H}), 3.35$ $(\mathrm{s}, 3 \mathrm{H}) ;{ }^{13} \mathrm{C}-\mathrm{NMR}\left(125 \mathrm{MHz}, \mathrm{CDCl}_{3}\right) \delta 170.1,168.6$, $161.6,142.5,131.2,131.0,130.7,130.2,130.0,129.1$, 122.5, 113.7, 56.7, 55.4, 34.7; LRMS (FAB) m/e 315 $(\mathrm{M}+\mathrm{H})^{+}$; HRMS (FAB) $m / e 315.0900\left[(\mathrm{M}+\mathrm{H})^{+}\right.$, calcd for $\mathrm{C}_{17} \mathrm{H}_{16} \mathrm{O}_{2} \mathrm{~N}_{2} \mathrm{Cl}$ : 315.0894].

\section{Benzodiazepine enolate alkylation}

\section{Method A}

To a solution of 4 (15 g, $48 \mathrm{mmol})$ in $200 \mathrm{~mL}$ of THF at $-78{ }^{\circ} \mathrm{C}$ was added $122 \mathrm{~mL}$ of $0.59 \mathrm{M}$ potassium tert-butoxide in THF. After $10 \mathrm{~min}$, a solution of 2-(bromomethyl)naphthalene $(13.7 \mathrm{~g}, 62 \mathrm{mmol})$ in THF $(20 \mathrm{~mL})$ was added by cannula. The solution was stirred for $1 \mathrm{~h}$ and quenched with $100 \mathrm{~mL}$ of saturated $\mathrm{NH}_{4} \mathrm{Cl}$ solution. The aqueous layer was extracted with ethyl acetate (3 x 150 $\mathrm{mL}$ ), and the combined organic layer was washed with water, brine, dried over sodium sulfate, and concentrated in vacuo. The residue was recrystallized from methanol to provide $16.8 \mathrm{~g}$ of $\mathbf{5 a}(78 \%)$ as a white solid.

\section{Method B}

To a solution of $4(100 \mathrm{mg}, 0.32 \mathrm{mmol})$ in $2.0 \mathrm{~mL}$ of THF at $-20{ }^{\circ} \mathrm{C}$ was added $0.83 \mathrm{~mL}$ of $0.5 \mathrm{M}$ potassium hexamethyldisilazide (KHMDS) in toluene. The solution was allowed to warm to $-5{ }^{\circ} \mathrm{C}$ and a solution of alkyl bromide $(0.42 \mathrm{mmol})$ in THF $(1 \mathrm{~mL})$ was added by cannula. After stirring overnight, the solution was quenched with saturated $\mathrm{NH}_{4} \mathrm{Cl}$ solution and then the work-up procedure described for Method A was followed.

7-Chloro-5-(4-methoxyphenyl)-1-methyl-3-(2-naphthylmet
hyl)-1,4-benzodiazepin-2-one (5a)

Mp 98-100 ${ }^{\circ} \mathrm{C} .{ }^{1} \mathrm{H}-\mathrm{NMR}\left(500 \mathrm{MHz}, \mathrm{CDCl}_{3}\right) \delta$ 7.75$7.85(\mathrm{~m}, 4 \mathrm{H}), 7.39-7.55(\mathrm{~m}, 6 \mathrm{H}), 7.26(\mathrm{dd}, J=13,6.5 \mathrm{~Hz}$, $2 \mathrm{H}), 6.91(\mathrm{~d}, J=8.5 \mathrm{~Hz}, 2 \mathrm{H}), 3.85(\mathrm{~s}, 3 \mathrm{H}), 3.70-3.89(\mathrm{~m}$, $3 \mathrm{H}), 3.41$ (s, 3H). ${ }^{13} \mathrm{C}-\mathrm{NMR}\left(125 \mathrm{MHz}, \mathrm{CDCl}_{3}\right) ; \delta 170.2$, $166.3,161.6,142.2,136.9,133.5,132.1,131.3,131.2$, $130.7,130.5,129.8,129.1,128.6,128.2$, 127.54, 127.49, 125.8, 125.2, 122.7, 113.7, 65.2, 55.4, 38.2, 35.2. HRMS (FAB) $m / e$ 455.1526 [(M+H) ${ }^{+}$, calcd for $\mathrm{C}_{28} \mathrm{H}_{24} \mathrm{O}_{2} \mathrm{~N}_{2} \mathrm{Cl}$ : 455.1528].

7-Chloro-5-(4-methoxyphenyl)-1-methyl-3-benzyl-1,4-ben zodiazepin-2-one $(\mathbf{5 b})$

Mp $194{ }^{\circ} \mathrm{C} .{ }^{1} \mathrm{H}-\mathrm{NMR}\left(400 \mathrm{MHz}, \mathrm{CDCl}_{3}\right) \delta 7.51(\mathrm{~d}, J=$ $8.9 \mathrm{~Hz}, 2 \mathrm{H}), 7.41-7.51(\mathrm{~m}, 1 \mathrm{H}), 7.13-7.40(\mathrm{~m}, 7 \mathrm{H}), 6.90(\mathrm{~d}$, $J=8.9 \mathrm{~Hz}, 2 \mathrm{H}), 3.83(\mathrm{~s}, 3 \mathrm{H}), 3.73(\mathrm{t}, J=6.3 \mathrm{~Hz}, 1 \mathrm{H})$, 3.55-3.69 (m, 2H), 3.39 (s, 3H). ${ }^{13} \mathrm{C}-\mathrm{NMR}(100 \mathrm{MHz}$, $\left.\mathrm{CDCl}_{3}\right) \delta 170.3,166.2,161.6,142.2,139.4,131.3131 .2$, $130.8,130.5,129.9,129.9,129.1,128.2,126.2,122.8$, 
113.8, 65.1, 55.4, 38.2, 35.2. HRMS (FAB) m/e 405.1370 $\left[(\mathrm{M}+\mathrm{H})^{+}\right.$, calcd for $\mathrm{C}_{24} \mathrm{H}_{22} \mathrm{O}_{2} \mathrm{~N}_{2} \mathrm{Cl}$ : 405.1368].

7-Chloro-5-(4-methoxyphenyl)-1-methyl-3-methyl-1,4-ben zodiazepin-2-one (5c)

Mp 106-108 ${ }^{\circ} \mathrm{C} .{ }^{1} \mathrm{H}-\mathrm{NMR}\left(400 \mathrm{MHz}, \mathrm{CDCl}_{3}\right) \delta 7.52(\mathrm{~d}$, $J=8.7 \mathrm{~Hz}, 2 \mathrm{H}), 7.41-7.51(\mathrm{~m}, 1 \mathrm{H}), 7.21-7.38(\mathrm{~m}, 2 \mathrm{H}), 6.88$ $(\mathrm{d}, J=8.7 \mathrm{~Hz}, 2 \mathrm{H}), 3.81(\mathrm{~s}, 3 \mathrm{H}), 3.65(\mathrm{q}, J=6.4 \mathrm{~Hz}, 1 \mathrm{H})$, $3.36(\mathrm{~s}, 3 \mathrm{H}), 1.67$ (d, $J=6.4 \mathrm{~Hz}, 3 \mathrm{H}) .{ }^{13} \mathrm{C}-\mathrm{NMR}(100 \mathrm{MHz}$, $\left.\mathrm{CDCl}_{3}\right) \delta 171.4,166.1,161.6,142.3,131.3,131.1,130.8$, 129.7, 129.0, 122.6, 113.7, 58.6, 55.4, 35.1, 17.5. HRMS (FAB) $m / e 329.1057\left[(\mathrm{M}+\mathrm{H})^{+}\right.$, calcd for $\mathrm{C}_{18} \mathrm{H}_{18} \mathrm{O}_{2} \mathrm{~N}_{2} \mathrm{Cl}$ : 329.1065].

7-Chloro-5-(4-methoxyphenyl)-1-methyl-3-allyl-1,4-benzo diazepin-2-one (5d)

Mp $152{ }^{\circ} \mathrm{C} .{ }^{1} \mathrm{H}-\mathrm{NMR}\left(400 \mathrm{MHz}, \mathrm{CDCl}_{3}\right) \delta 7.54(\mathrm{~d}, J=$ $8.7 \mathrm{~Hz}, 2 \mathrm{H}), 7.42-7.53(\mathrm{~m}, 1 \mathrm{H}), 7.0-7.35(\mathrm{~m}, 2 \mathrm{H}), 6.89(\mathrm{~d}$, $J=8.7 \mathrm{~Hz}, 2 \mathrm{H}), 5.89-6.03(\mathrm{~m}, 1 \mathrm{H}), 5.14(\mathrm{~d}, J=17.2 \mathrm{~Hz}$, $1 \mathrm{H}), 5.05(\mathrm{~d}, J=10.2 \mathrm{~Hz}, 1 \mathrm{H}) 3.82(\mathrm{~s}, 3 \mathrm{H}), 3.54(\mathrm{t}, J=6.8$ $\mathrm{Hz}, 1 \mathrm{H}), 3.37$ (s, 3H), 2.96 (t, $J=6.8 \mathrm{~Hz}, 2 \mathrm{H}) .{ }^{13} \mathrm{C}-\mathrm{NMR}$ $\left(100 \mathrm{MHz}, \mathrm{CDCl}_{3}\right) \delta 170.3,166.2,161.6,142.3,135.6$, $131.3131 .2,130.8,130.6,129.8,129.1,122.7,117.0$, 113.7, 63.3, 55.4, 36.3, 35.1. HRMS (FAB) m/e 355.1213 $\left[(\mathrm{M}+\mathrm{H})^{+}\right.$, calcd for $\left.\mathrm{C}_{20} \mathrm{H}_{20} \mathrm{O}_{2} \mathrm{~N}_{2} \mathrm{Cl}: 355.1212\right]$.

7-Chloro-5-(4-methoxyphenyl)-1-methyl-3-ethyl-1,4-benzo diazepin-2-one (5e)

Mp 108-110 ${ }^{\circ} \mathrm{C} .{ }^{1} \mathrm{H}-\mathrm{NMR}\left(400 \mathrm{MHz}, \mathrm{CDCl}_{3}\right) \delta 7.54(\mathrm{~d}$, $J=8.8 \mathrm{~Hz}, 2 \mathrm{H}), 7.48(\mathrm{dd}, J=8.8 \mathrm{~Hz}, 2.5 \mathrm{~Hz}, 1 \mathrm{H}), 7.25-7.39$ $(\mathrm{m}, 2 \mathrm{H}), 6.89(\mathrm{~d}, J=8.8 \mathrm{~Hz}, 2 \mathrm{H}), 3.83(\mathrm{~s}, 3 \mathrm{H}), 3.37(\mathrm{~s}, 3 \mathrm{H})$ 3.32-3.40 (m, 1H) 2.15-2.30 (m, 2H) $1.01(\mathrm{t}, J=7.4 \mathrm{~Hz}$, $3 \mathrm{H}) .{ }^{13} \mathrm{C}-\mathrm{NMR}\left(100 \mathrm{MHz}, \mathrm{CDCl}_{3}\right) \delta 170.7,166.3,161.6$, 142.4, 131.2 131.1, 130.9, 130.7, 129.8, 129.0, 122.7, 113.7, 64.8, 55.4, 35.0, 24.9, 10.7. HRMS (FAB) $\mathrm{m} / \mathrm{e}$ $343.1213\left[(\mathrm{M}+\mathrm{H})^{+}\right.$, calcd for $\mathrm{C}_{19} \mathrm{H}_{20} \mathrm{O}_{2} \mathrm{~N}_{2} \mathrm{Cl}$ : 343.1209].

7-Chloro-5-(4-methoxyphenyl)-1-methyl-3-isobutyl-1,4-be nzodiazepin-2-one (5f)

Mp 185-186 ${ }^{\circ} \mathrm{C} .{ }^{1} \mathrm{H}-\mathrm{NMR}\left(400 \mathrm{MHz}, \mathrm{CDCl}_{3}\right) \delta 7.53(\mathrm{~d}$, $J=8.8 \mathrm{~Hz}, 2 \mathrm{H}), 7.48-7.53(\mathrm{~m}, 1 \mathrm{H}), 7.32(\mathrm{~d}, J=2.4 \mathrm{~Hz}, 1 \mathrm{H})$, 7.24-7.30 (m, 1H), $6.90(\mathrm{~d}, J=8.8 \mathrm{~Hz}, 2 \mathrm{H}), 3.84(\mathrm{~s}, 3 \mathrm{H})$, 3.50-3.59 (m, 1H), $3.38(\mathrm{~s}, 3 \mathrm{H}), 2.23-2.32(\mathrm{~m}, 1 \mathrm{H}), 1.89-$ $2.00(\mathrm{~m}, 2 \mathrm{H}), 0.98(\mathrm{~d}, J=6.4 \mathrm{~Hz}, 3 \mathrm{H}), 0.79(\mathrm{~d}, J=6.4 \mathrm{~Hz}$, $3 \mathrm{H}) .{ }^{13} \mathrm{C}-\mathrm{NMR}\left(100 \mathrm{MHz}, \mathrm{CDCl}_{3}\right) \delta 170.9,166.2,161.2$, $142.4,131.3,131.1131 .0,130.6,129.7,129.0,122.6$, 113.7, 61.4, 55.4, 40.3, 35.1, 24.6, 23.5, 22.0. HRMS (FAB) $m / e 371.1526\left[(\mathrm{M}+\mathrm{H})^{+}\right.$, calcd for $\mathrm{C}_{21} \mathrm{H}_{24} \mathrm{O}_{2} \mathrm{~N}_{2} \mathrm{Cl}$ : 371.1528].
7-Chloro-5-(4-methoxyphenyl)-1-methyl-3-(methylcyclohe $x y l)$-1,4-benzodiazepin-2-one (5g)

Mp 155-156 ${ }^{\circ} \mathrm{C} .{ }^{1} \mathrm{H}-\mathrm{NMR}\left(400 \mathrm{MHz}, \mathrm{CDCl}_{3}\right) \delta 7.52(\mathrm{~d}$, $J=8.9 \mathrm{~Hz}, 2 \mathrm{H}), 7.45-7.53(\mathrm{~m}, 1 \mathrm{H}), 7.32(\mathrm{~d}, J=2.4 \mathrm{~Hz}, 1 \mathrm{H})$, 7.22-7.30 (m, 1H), $6.89(\mathrm{~d}, J=8.9 \mathrm{~Hz}, 2 \mathrm{H}), 3.82(\mathrm{~s}, 3 \mathrm{H})$, 3.57 (dd, $J=9.0 \mathrm{~Hz}, 5.2 \mathrm{~Hz}, 1 \mathrm{H}), 3.37$ (s, $3 \mathrm{H}), 2.17-2.29$ $(\mathrm{m}, 1 \mathrm{H}), 1.9-2.02(\mathrm{~m}, 1 \mathrm{H}), 1.45-1.80(\mathrm{~m}, 5 \mathrm{H}), 0.9-1.31(\mathrm{~m}$, $6 \mathrm{H}) .{ }^{13} \mathrm{C}-\mathrm{NMR}\left(100 \mathrm{MHz}, \mathrm{CDCl}_{3}\right) \delta 170.9,166.1,161.5$, $142.4,131.3,131.1131 .0,130.6,129.7,129.0,122.7$, 113.7, 60.8, 55.4, 39.0, 35.1, 34.1, 32.7, 26.6, 26.4, 26.3. HRMS (FAB) $m / e$ 411.1839 $\left[(\mathrm{M}+\mathrm{H})^{+}\right.$, calcd for $\mathrm{C}_{24} \mathrm{H}_{28} \mathrm{O}_{2} \mathrm{~N}_{2} \mathrm{Cl}$ : 411.1836].

7-Chloro-5-(4-hydroxyphenyl)-1-methyl-3-(2-naphthylmet hyl)-1,4-benzodiazepin-2-one

To a solution of $\mathbf{5 a}(17 \mathrm{~g}, 37 \mathrm{mmol})$ in ethanethiol (300 $\mathrm{mL}$ ) at $0{ }^{\circ} \mathrm{C}$ was added $222 \mathrm{~mL}$ of $1 \mathrm{M}$ aluminum bromide in dibromomethane. The mixture was allowed to warm to room temperature and stirred for $3 \mathrm{~h}$. The pale yellow solution was poured into about $100 \mathrm{~g}$ of ice in one portion and the precipitate was filtered. The white powder was partitioned between $200 \mathrm{~mL}$ of $1 \mathrm{M} \mathrm{HCl}$ solution and 400 $\mathrm{mL}$ of methanol- $\mathrm{CHCl}_{3}(1: 1)$. The mixture was concentrated in vacuo. The aqueous layer was extracted with ethyl acetate $(3 \times 200 \mathrm{~mL})$, the combined extracts were washed with water, brine, dried over sodium sulfate, and concentrated in vacuo. The residue was recrystallized from methanol to furnish $14 \mathrm{~g}$ of $1(86 \%)$ as a white solid: $\mathrm{mp}$ $153-155{ }^{\circ} \mathrm{C}$; ${ }^{1} \mathrm{H}-\mathrm{NMR}\left(400 \mathrm{MHz}, \mathrm{DMSO}-d_{6}\right) \delta 9.98(\mathrm{~s}$, $1 \mathrm{H}), 7.71-7.83(\mathrm{~m}, 4 \mathrm{H}), 7.32-7.63(\mathrm{~m}, 5 \mathrm{H}), 7.33(\mathrm{~d}, J=8.5$ $\mathrm{Hz}, 2 \mathrm{H}), 7.14(\mathrm{~d}, J=2.2 \mathrm{~Hz}, 1 \mathrm{H}), 6.78(\mathrm{~d}, J=8.5 \mathrm{~Hz}, 2 \mathrm{H})$, 3.77 (br t, $J=7.2 \mathrm{~Hz}, 1 \mathrm{H}$ ), 3.47-3.51 (m, 2H), 3.29 (s, 3H); ${ }^{13} \mathrm{C}-\mathrm{NMR}\left(100 \mathrm{MHz}, \mathrm{DMSO}-d_{6}\right) \delta 170.1,166.0,160.3$, $142.5,137.3,133.5,132.1,131.8,131.4,130.4,129.2$, 129.1, 129.0, 128.34, 128.25, 127.9, 127.81, 127.78, 126.3, 125.7, 124.4, 115.6, 64.9, 38.3, 35.1; LRMS (FAB) m/e 441 $(\mathrm{M}+\mathrm{H})^{+}$; HRMS (FAB) $m / e 441.1370\left[(\mathrm{M}+\mathrm{H})^{+}\right.$, calcd for $\mathrm{C}_{27} \mathrm{H}_{22} \mathrm{O}_{2} \mathrm{~N}_{2} \mathrm{Cl}$ : 441.1379].

\section{Acknowledgment}

We gratefully acknowledge the National Institutes of Health (GM50353) for support of this work.

\section{References}

1. Sternbach, L.H. J. Med. Chem. 1979, $22,1$.

2. Bock, M.G.; Dipardo, R.M.; Evans, B.E.; Rittle, K.E.; Whitter, W.L.; Veber, D.F.; Anderson, P.S.; Freidinger, R.M. J. Med. Chem. 1989, 32, 13.

3. Romer, D.; Buschler, H.H.; Hill, R.C.; Maurer, R.; Petcher, T.J.; Zeugner, H.; Benson, W.; Finner, E.; Milkowski, W.; Thies, P.W. Nature 1982, 298, 759.

4. Korneki, E.; Erlich, Y.H.; Lenox, R.H. Science 1984, 226, 1454. 
5.Hsu, M.C.; Schutt, A.D.; Holly, M.; Slice, L.W.; Sherman, M.I.; Richman, D.D.; Potash, J.M.; Volsky, D.F. Science 1991, 254, 1799.

6. Pauwels, R.; Andries, K.; Desmyter, J.; Schols, D.; Kukla, M.J.; Breslin, H.; Raeymaeckers, A.; Van, G. Nature 1990, 343, 470.

7. James, G.L.; Goldstein, J.L.; Brown, M.S.; Rawson, T.E.; Somers, T.C.; Medowell, R.S.; Crowley, C.W.; Lucas, B.K.; Levinson, A.D.; Marsters, J.C. Science 1993, 260, 1937.

8. Bunin, B.A.; Ellman, J.A. J. Am. Chem. Soc. 1992, 114, 10997.

9. Bunin, B.A.; Plunkett, M.J.; Ellman, J.A. Proc. Natl. Acad. Sci. U.S.A. 1994, 6, 4708.

10. Plunkett, M.J.; Ellman, J.A. J. Am. Chem. Soc. 1995, 117, 3306.

11. Bunin, B.A.; Plunkett, M.J.; Ellman, J.A. Methods Enzymol. 1995, 267, 448.

12. Boojamra, C.G.; Burow, K.M.; Ellman, J.A. J. Org. Chem. 1997, 62, 1240.
13. Stevens, S.Y.; Bunin, B.A.; Plunkett, M.J.; Swanson, P.C.; Ellman, J.A.; Glick, G.D. J. Am. Chem. Soc. 1996, $118,10650$.

14. Hahn, B.H. N. Eng. J. Med. 1998, 338, 1359.

15. Bock, M.G.; Dipardo, R.M.; Evans, B.E.; Rittle, K.E.; Veber, D.F.; Freidinger, R.M.; Hirshfield, J.; Springer, J.P. J. Org. Chem. 1987, 52, 3232.

16. Fryer, R.I.; Earley, J.V.; Sternbach, L.H. J. Org. Chem. 1969, 34, 649.

17. Reitter, B.E.; Sachdeva, Y.P.; Wolfe, J.F. J. Org. Chem. 1981, 46, 3945.

18. Butcher, J.W.; Liverton, N.J.; Selnick, H.G.; Elliot, J.; Smith, G.R. Tetrahedron Lett. 1996, 37, 6685.

19. Davis, R.B.; Pizzini, L.C. J. Org. Chem. 1960, 25, 1884.

20. Sternbach, L.H.; Fryer, R.I.; Metlesics, W.; Reeder, E.; Sach, G.; Saucy, G.; Stempel, A. J. Org. Chem. 1962, 27, 3788.

21. Node, M.; Nishide, K.; Fujita, E. J. Org. Chem. 1980, $45,4275$. 